\title{
Is Long-Term Therapy With Thiopurines Effective for Maintaining Remission in Patients With Moderate-To-Severe Ulcerative Colitis?
}

\author{
Seong Ran Jeon ${ }^{1}$, Won Ho Kim² \\ Departments of Internal Medicine, Soonchunhyang University College of Medicine ${ }^{1}$, Yonsei University College of Medicine ${ }^{2}$, Seoul, Korea

\section{Article: Efficacy and Safety of Long-Term Thiopurine Maintenance Treatment in Japanese Patients With Ulcerative Colitis (Intest Res 2015;13:250-258)}

The thiopurines, azathioprine and 6-mercaptopurine, have been in clinical use for 50 years. Prospective studies and meta-analyses suggest that thiopurines are effective for the maintenance of remission in UC. ${ }^{1,2}$ By maintaining remission, hospitalization and colectomy rates can be reduced, and long-term complications prevented. However, in the biologic era, the long-term efficacy of thiopurine needs to be better understood.

In this issue of Intestinal Research, Yamada et al. tried to assess the long-term efficacy and safety of maintenance treatment with thiopurines in UC. ${ }^{3}$ They enrolled a total of 59 bio-naive patients who maintained remission by treatment with thiopurine and 5 -aminosalicylate after induction. The cumulative remission-maintenance rates at 24,48 , and 84 months were reported as approximately $69 \%, 55 \%$, and $44 \%$, respectively. Recent meta-analyses of trial data revealed remission maintenance rates of $56-76 \%$ during thiopurine treatment. ${ }^{1,2}$ The remission maintenance rate of Yamada et al. was similar to that of meta-analyses, considering that the mean follow-up period of these studies was shorter. Yamada et al. also demonstrated that high-dose corticosteroid treatment $(\geq 40 \mathrm{mg} /$ day) was a negative predictive factor for

Received May 1, 2015. Revised May 2, 2015. Accepted May 2, 2015. Correspondence to Won Ho Kim, Department of Internal Medicine and Institute of Gastroenterology, Yonsei University College of Medicine, 50-1 Yonsei-ro, Seodaemun-gu, Seoul 120-752, Korea. Tel: +82-2-2228-1963, Fax:+82-2-365-2125, E-mail: kimwonho@yuhs.ac

Financial support: None. Conflict of interest: None. maintenance treatment with thiopurine. This could be inferred from the fact that other maintenance treatments such as biologics should be considered in refractory UC patients treated with high-dose corticosteroids.

Mucosal healing (MH) is becoming more widely accepted as a relevant outcome marker for UC. In a Norwegian population-based cohort study examining effects between the presence or absence of MH and long-term outcomes, $\mathrm{MH}$ at 1 year was confirmed in $50 \%$ of UC patients, and the colectomy rate at 5 years was $1.7 \%$. MH at 1 year significantly decreased the need for colectomy at 5 years, ${ }^{4}$ and this association was significant after adjusting for factors that may influence colectomy, such as age, smoking, education level, and disease extent. Therefore, the previous study provides evidence that $\mathrm{MH}$ is strongly associated with the clinical outcomes of UC. Yamada et al. reported MH rates of $>60 \%$ at 26.8 months, and a colectomy rate of $3.6 \%$. Compared to that of Western studies, this result was not significantly different. However, only two-thirds of study patients underwent colonoscopy to evaluate $\mathrm{MH}$ and the time interval between initiating thiopurine therapy and performing the colonoscopy to assess MH was different in each patient due to the retrospective design of the study. Therefore, the results of Yamada et al. should be interpreted while considering the limitations of the study, such as the small number of patients included and the lack of adjustment for potential confounding factors.

Efficacy must be balanced against safety and tolerability. In the Yamada et al. study, the median dose of thiopurine was $50 \mathrm{mg}$. Traditionally, the dose-escalating approach is used in

๑ Copyright 2015. Korean Association for the Study of Intestinal Diseases. All rights reserved.

This is an Open Access article distributed under the terms of the Creative Commons Attribution Non-Commercial License (http://creativecommons.org/licenses/by-nc/4.0)

which permits unrestricted non-commercial use, distribution, and reproduction in any medium, provided the original work is properly cited. 
East Asian patients. ${ }^{5,6}$ Several trials, including this study, have suggested that lower doses of azathioprine or 6-mercaptopurine may be effective and safe for the treatment of UC patients, ${ }^{7,8}$ contrary to the results of Western studies. According to previous studies, approximately $10-28 \%$ of patients reported adverse events (AEs), and $25.6 \%$ of patients had to discontinue thiopurine therapy due to AEs. ${ }^{9,10}$ A similar rate of AEs (28.8\%) was reported in the study by Yamada et al. In this study, the discontinuation rate due to AEs was reported as low as $10.2 \%$, probably due to low thiopurine dose they used. Although there were no severe AEs such as lymphoma and non-melanoma skin cancer in the Yamada et al. study, and the absolute risks of these malignancies remain low, the risks and benefits of continuing or ceasing thiopurine therapy should be weighed for each individual patient.

Despite the fact that clinical trials (including the Yamada et al. study) concerning the use of thiopurines for UC have been heterogeneous in design, the evidence is sufficient to conclude that thiopurines are effective for maintenance of remission.

\section{REFERENCES}

1. Gisbert JP, Linares PM, McNicholl AG, Maté J, Gomollón F. Meta-analysis: the efficacy of azathioprine and mercaptopurine in ulcerative colitis. Aliment Pharmacol Ther 2009;30:126-137.

2. Timmer A, McDonald JW, Macdonald JK. Azathioprine and 6-mercaptopurine for maintenance of remission in ulcerative colitis. Cochrane Database Syst Rev doi: 10.1002/14651858. CD000478.pub2. Published online 24 January 2007.
3. Yamada S, Yoshino T, Matsuura M, et al. Efficacy and safety of long-term thiopurine maintenance treatment in Japanese patients with ulcerative colitis. Intest Res 2015;13:250-258.

4. Frøslie KF, Jahnsen J, Moum BA, Vatn MH, IBSEN Group. Mucosal healing in inflammatory bowel disease: results from a Norwegian population-based cohort. Gastroenterology 2007;133:412-422.

5. Kim JH, Cheon JH, Kim TI, Kim WH. A survey of actual clinical practice patterns in the treatment of inflammatory bowel disease in Korea. Intest Res 2009;7:79-85.

6. Chande N, Tsoulis DJ, MacDonald JK. Azathioprine or 6-mercaptopurine for induction of remission in Crohn's disease. Cochrane Database Syst Rev doi: 10.1002/14651858.CD000545. pub4. Published online 30 April 2013.

7. Park SK, Yang SK, Ye BD, et al. The long-term efficacy of azathioprine in steroid-dependent ulcerative colitis. Scand J Gastroenterol 2013;48:1386-1393.

8. Hibi T, Naganuma M, Kitahora T, Kinjyo F, Shimoyama T. Lowdose azathioprine is effective and safe for maintenance of remission in patients with ulcerative colitis. J Gastroenterol 2003;38:740-746.

9. Chaparro M, Ordás I, Cabré E, et al. Safety of thiopurine therapy in inflammatory bowel disease: long-term follow-up study of 3931 patients. Inflamm Bowel Dis 2013;19:1404-1410.

10. Gearry RB, Barclay ML, Burt MJ, Collett JA, Chapman BA. Thiopurine drug adverse effects in a population of New Zealand patients with inflammatory bowel disease. Pharmacoepidemiol Drug Saf 2004;13:563-567. 